\title{
Caracterisation Sedimentologique Et Hydrodynamique Des Formations Sableuses Du Miocene Inferieur De La Region D'eboinda (Sud-Est De La Cote d'Ivoire)
}

\author{
Kizito Katel Kahou Toé Bi \\ N'goran Jean-Paul Yao
}

Université Felix-Houphouët-Boigny de cocody, UFR des Sciences de la

Terreet des Ressources Minières, Abidjan, Côte d'Ivoire

Touvalé Marcel Kessé

Institut National Polytechnique Felix-Houphouët-Boigny,

Yamoussoukro, Côte d'Ivoire

\section{Zéli Bruno Digbéhi}

Université Felix-Houphouët-Boigny de cocody, UFR des Sciences de la

Terre et des Ressources Minières, Abidjan, Côte d'Ivoire

Abstract

Five hundred and five (505) cuttings samples of 51 survey (of $10 \mathrm{~m}$ depth maximum) drilled in Eboinda area (South-western of Côte d'Ivoire) were subject to lithological, mineralogical an granulometry studies in order to clarify the nature of sediments and factors and processes involved during their transport and deposition. The "Surfer" software helps to map the formations.

The results indicate:

- lithologically:, six mains facies were defined ; sandy clays with gravel, clayey sand, sands, siltyey clay, sandy clays and clay. Sandy sediments were mapped to north and clayed one in south of the study area. Most of studied sands were interpreted as river and coastal dune deposits. Many minerals were described such as sphene, zircon, tourmaline, ilmenite, hematite, apatite, limonite, sillimanite, chromite, muscovite, garnet, chlorite, rutile, goethite, quartz and feldspar. The clay fraction is composed by kaolinite, quartz, apatite, gypsum, goethite and halloysite;

- hydrodynamically: the surfacecreep is the main mode of transport expressed within units III and VI. While saltation characterizes sand units IV and V. Predominance of rounded forms and sub-rounded features of quartz grains suggests a transport in aqueous zone over a long distance. In 
the other hand the sands of units VI and III are essentially sub-angular, suggesting a close generating source. The blunt and shiny aspect of most sands grains encountered, confirm the mode of transport in aqueous zone.

Keywords: Sedimentology, sedimentary hydrodynamics, Ivory Coast Basin

\section{Résumé}

Cinquant-cinq (505) échantillons de déblais issus de 51 sondages peu profonds (maximum $10 \mathrm{~m}$ ) réalisés dans la région d'Eboinda (Sud-Est de la Côte d'Ivoire) ont fait l'objet d'études lithologique, minéralogique et granulométrique en vue de préciser la nature des sédiments ainsi que les facteurs et les phénomènes intervenus au cours de leur transport et leur dépôt. A l'aide du logiciel « Surfer », les différentes formations ont été cartographiées. Les résultats obtenus indiquent:

- au plan lithologique, six faciès notamment ; l'argile sableuse gavuleuse, le sable argileux, le sable, l'argile sableuse, l'argile silteuse et l'argile. Les sédiments sableux sont cartographiés au Nord du domaine d'étude alors que, les sédiments argileux sont localisés Sud. L'essentiel des sables étudiés est interprété comme des dépôts de type rivière et dune côtière. Le cortège minéralogique comporte de nombreux minéraux tels que : le sphène, le zircon, la tourmaline, l'ilménite, l'hématite, l'apatite, la limonite, la sillimanite, la chromite, la muscovite, la grenat, la chlorite, le rutile, la goethite, le quartz et les feldspaths. La fraction argileuse majoritairement formée de kaolinite comporte aussi du quartz, de l'apatite, du gypse, de la goethite et de la halloysite;

- au plan hydrodynamique, le roulement est le mode de transport dominant des sédiments des unités III et VI. contrairement aux unités IV et V où domine la saltation. L'étude morphoscopique révèle une prédominance des formes arrondies et sub-arrondies suggérant ainsi un transport en milieu aqueux sur une longue distance. Par contre les sables des unités VI et III sont essentiellement sub-anguleux, évoquant une source génératrice proche. L'aspect émoussé luisant de la plupart des grains rencontrés, confirme le mode de transport en milieux aqueux.

Mots Clés : Sédimentologie, hydrodynamique sédimentaire, bassin de Côte d'Ivoire

\section{Introduction}

La zone d'étude est située dans le Sud-Est de la Côte d'Ivoire (région d'Aboisso, Figure 1). Elle occupe le Sud de la faille des lagunes avec un réseau hydrographique dominé par les lagunes Aby, Tendo, Ehy. Les travaux de Dian (1977), Dioulo (2009) et SODEMI (2011) qui ont révélé et 
évalué le potentiel des indices de phosphates. Par ailleurs, les investigations de PETROCI et BEICIP (1990) et Assalé (2013) ont indiqué la présence des niveaux bitumineux aux affleurements de Samo et d'Eboinda. Toutes ces données restent limitées à la connaissance quantitative du phosphate et du potentiel pétrolier. Ainsi, les caractéristiques géologiques portant sur l'environnement des dépôts du Miocène (notamment les données sédimentologiques plus précises) demeurent mal connues. C'est pourquoi, la nature des sédiments, leurs caractéristiques granulométriques et minéralogiques, leur origine et leur mode de déplacement sont étudiés. Les présents travaux donc visent à comprendre les processus sédimentaires qui ont gouverné ces dépôts au cours de cette époque.

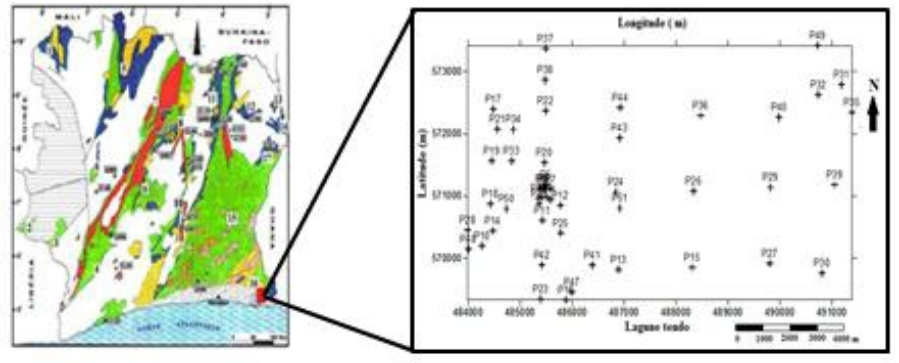

Fig. 1 : Situation géographique de la zone d'étude

\section{Matériel et Méthodes}

Au total 505 échantillons de sédiments ont été prélevés dans 51 puits d'hommes. Après une description lithologique sur le terrain, les échantillons ont été séchés à $50{ }^{\circ} \mathrm{C}$ à l'étuve puis tamisés par voie humide sur une maille de $40 \mu \mathrm{m}$. La fraction grossière $(>40 \mu \mathrm{m})$ a fait l'objet d'une analyse granulométrique à sec sur une colonne de 16 tamis (Vibrotechnik) après une élimination de la matière organique à l'eau oxygénée et des carbonates à l'acide chlorhydrique.

A partir des courbes cumulatives obtenues des indices granulométriques ont été déterminés selon la méthode de Folk et Ward (1957) et Friedman (1961). Le mode de transport des sédiments a été défini par la méthode de Visher (1969). Les milieux de dépôt ont été déterminés à partir des diagrammes So-Md et Md-Sk de Moiola et Weiser (1968).Ces diagrammes de dispersion distinguent les environnements de dépôt de dune continentale et dune côtière, du milieu de dépôt de rivière et de plage. La morphoscopie des grains de quartz a permis de déterminer l'usure et l'agent de transport de ceux-ci par la méthode de Cailleux (1947). Les cartes sédimentologiques et les cartes de positionnement ont été réalisées à l'aide des logiciels SURFER 8.0. 


\section{Résultats et Interpretation}

- Nature lithologique des unités rencontrées

La synthèse lithologique des sondages (Figure 2) indique une sédimentation dominée par six unités lithologiques inégalement réparties dans le domaine d'étude.

\section{- Unité I: l'argile}

Cette argile est grise à noire, tendre, silteuse et glauconieuse à rares passées de grès friables à grains subarrondis. Ces grains sont fins à moyens, parfois à grossiers et translucides. Quelques nodules noirs de 1 à $2 \mathrm{~cm}$, subarrondis dégagent une odeur de sulfure d'hydrogène. La pyrite et des moules de gastéropodes (tels que les genres Turritella et Pleurotomaria) sont présents. Cette unité est fortement imprégnée de bitume se présentant sous deux aspects (un aspect pâteux tendre à friable et un aspect consolidé ou solidifié). Cette unité repose dans la plupart des sondages sur une nappe phréatique.

\section{- Unité II : l'argile silteuse}

C'est une argile de couleur jaune, silteuse à odeur de sulfure d'hydrogène (un gaz toxique à odeur d'œuf pourri). Il y a également des débris carbonés, de la pyrite, la glauconite.

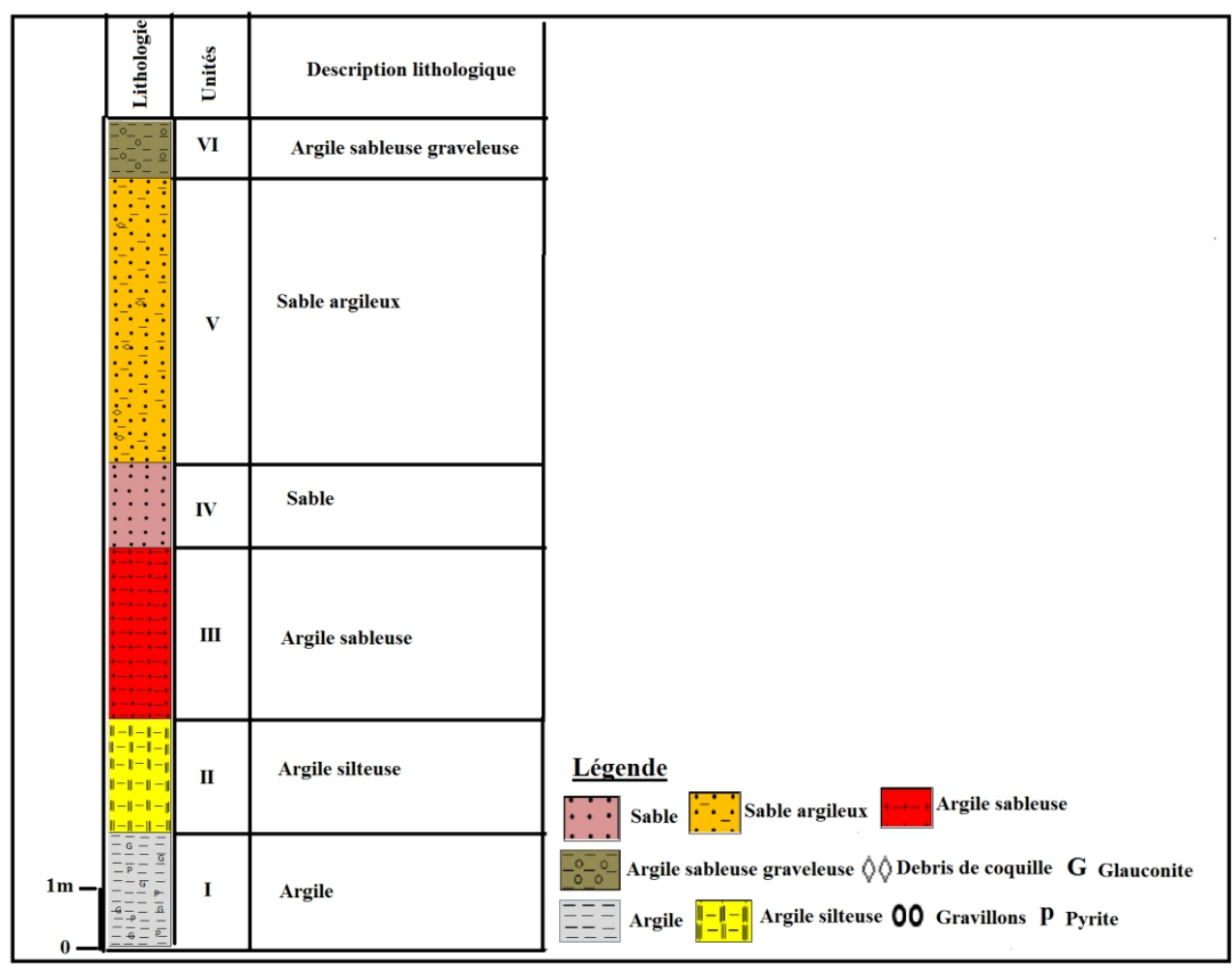

Fig. 2 : Synthèse lithologique des puits étudiés 
- Unité III: l’argile sableuse

Cette argile de couleur rougeâtre à bariolée, friable à tendre est faiblement micacée. La fraction sableuse comporte des grains translucides à rougeâtre, sub-arrondis à sub-anguleux, moyens à très fins. La glauconite, la pyrite et les débris de coquillers sont rares.

\section{- Unité IV : le sable}

Dans cette unité le sable est luisant, translucide, de couleur grise, jaune orangé, blanchâtre, sub-anguleux, sub-sphérique à sub-aplati. De taille moyenne à grossière.

\section{- Unité $\mathbf{V}$ : le sable argileux}

Ces sables grossiers à fins sont arrondis à sub-anguleux, translucides, rouge-orangé et laiteux par endroits. Ils sont relativement bien triés et contiennent de rares débris coquillers. La fraction argileuse est brunrougeâtre, friable. La pyrite y est très rare, contrairement aux paillettes de micas qui sont nombreuses. Comme dans l'unité 1 , aucun indice de bitume n'a été observé.

\section{- Unité VI : l'argile sableuse graveleuse}

Cette argile de couleur brune à noire est parfois oxydée, tendre à modérément ferme, friable à plastique. La fraction sableuse comporte des grains fins translucides, arrondis à sub-anguleux ainsi que la présence gravillons latéritiques, subarrondis à sub-anguleux.

\section{- Répartition spatiale des unités}

Les différentes cartes isopaches (Figures 3-8) ont permis d'estimer la variation de l'épaisseur des six formations rencontrées dans la zone d'étude.

\section{- $\quad$ Isopache de l'unité I ( fig 3)}

Cette unité se rencontre par endroits uniquement dans le Sud et le Sud-Ouest du périmètre d'étude. Les épaisseurs des sédiments ne dépassent pas les $5 \mathrm{~m}$.

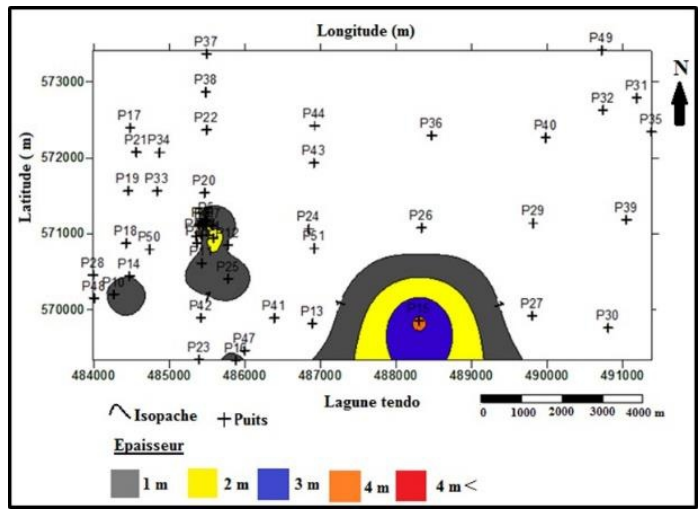

Fig. 3: Carte des isopaches de l'unité I 


\section{- $\quad$ Isopache de l'unité II ( fig 4)}

L'épaisseur de l'unité II de 1 à plus de $4 \mathrm{~m}$, les plus importantes sont comprises entre $3 \mathrm{~m}$ et $4 \mathrm{~m}$ dans la partie ouest de la zone d'étude. Au Sud et dans une moindre mesure au Nord-Est, les dépôts sont moins épais compris entre 1 et $2 \mathrm{~m}$.

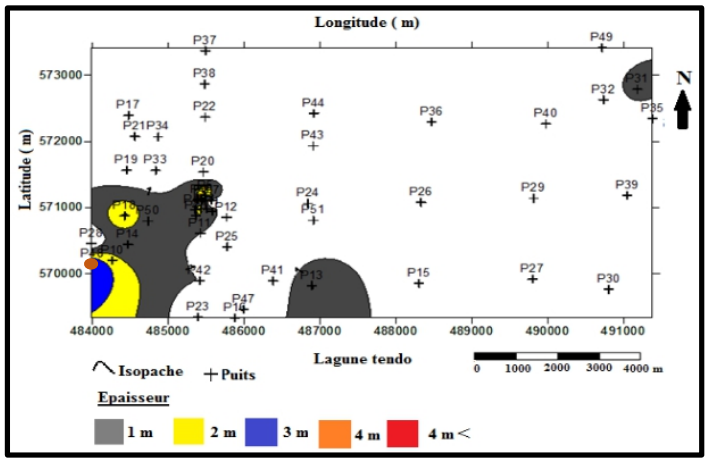

Fig. 4 : Carte des isopaches de l'unité II

\section{- $\quad$ Isopache de l'unité III (fig 5)}

La figure 5 présente une allure régulière, concentrique fermée vers le Sud-Ouest et ouverte vers la lagune Tendo (Sud). Ces isopaches montrent que les épaisseurs varient progressivement et ne dépassent généralement pas $7 \mathrm{~m}$, avec des valeurs les plus fortes se concentrent au Sud-Ouest.

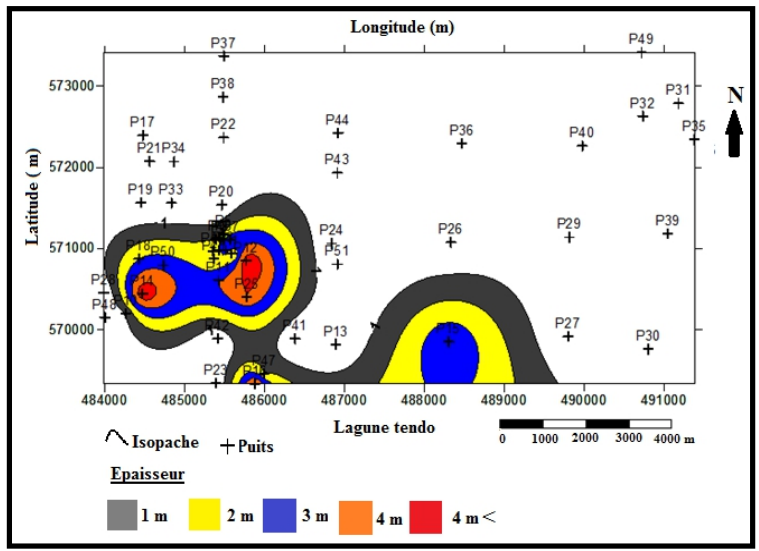

Fig. 5 : Carte des isopaches de l'unité III

\section{- Isopaches de l'unité IV (fig6)}

Elle montre des dépôts très localisés vers la lagune Tendo et peu épais, ne dépassant pas $5 \mathrm{~m}$ avec des isopaches régulières (allongées et circulaires). 


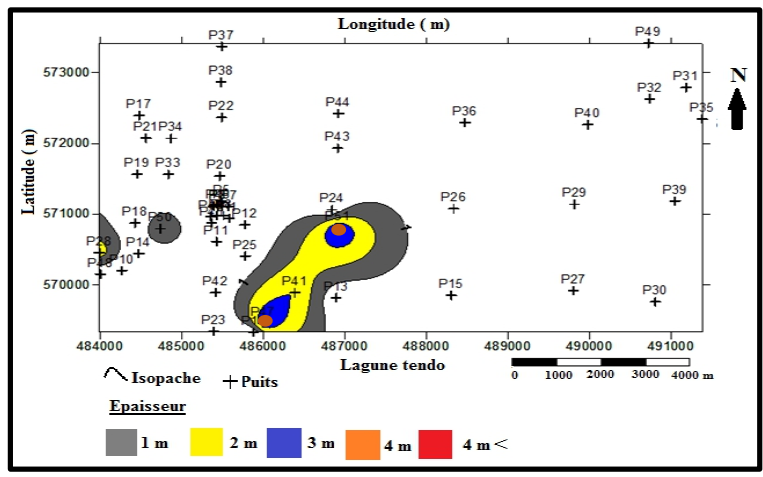

Fig. 6 : Carte des isopaches de l'unité IV

\section{- Isopache de l'unité V (fig 7)}

Elle est la plus représentée dont les puissances atteignent parfois 8 à 10 .

Elle est absente par endroits notamment dans le Sud-Ouest et le Sud.

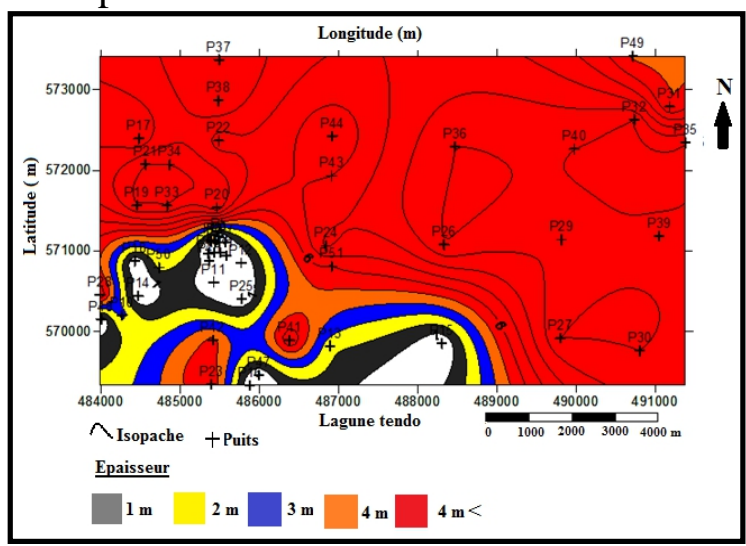

Fig. 7 : Carte des isopaches de l'unité V

\section{- $\quad$ Isopache de l'unité VI (fig 8)}

Cette carte montre des épaisseurs ne dépassant pas les $3 \mathrm{~m}$ dans une unité lithologique uniquement localisée au Sud-Ouest du périmètre d'étude

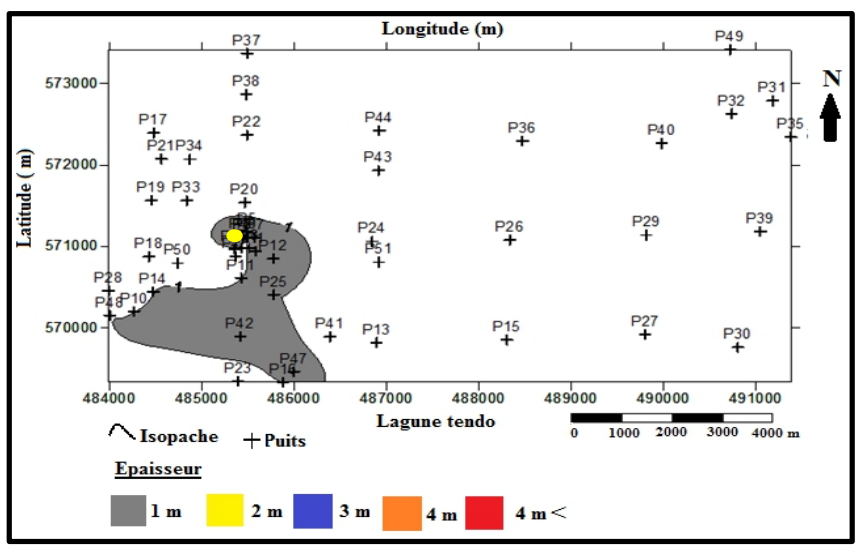

Fig. 8 : Carte des isopaches de l'unité VI du périmètre d'étude 
L'argile et le sable sont les seules lithologies des formations traversées dans les différents puits réalisés. Leur absence dans certains puits serait liée à une érosion différentielle. C'est le cas des unités I, II, III IV et VI dans la partie nord de la zone d'étude. A l'inverse, ces unités existent dans les zones supposées de faible degré érosif ou les paléo-chénaux. Les prépondérances des sédiments sableux plus grossiers sédiments dans la partie nord dénotent d'une forte énergie du palécourant par rapport aux dépôts plus argileux argileux dans la partie Sud. Ainsi, il s'en déduit le sens du paléocourant qui est orienté du Nord au Sud. Les gravillons localisés précisément entre les latitudes $(571000 \mathrm{~m}$ et $572000 \mathrm{~m})$ et les longitudes $(485000 \mathrm{~m}$ et $486000 \mathrm{~m}$ ) peuvent provenir des anciens fleuves ou rivières torrentiels ou de l'érosion des flancs des vallées. Ces gravillons latéritiques peuvent aussi correspondre au démantèlement du socle précambrien.

\section{Faciès granulométriques des sédiments (fig 9)}

L'analyse des courbes cumulatives semi-logarithmiques des sables étudiés montre deux types de faciès (logarithmique et sigmoïdal).

Le faciès logarithmique se rencontre dans les unités III (a) et VI (b). Il caractérise surtout un dépôt par excès de charge lors de la diminution de la vitesse de l'agent de transport.

Le faciès sigmoïdal observé dans les unités IV (c) et V (d), indique une réduction progressive de l'agent de transport. C'est une accumulation libre des sédiments transportés par roulement ou par saltation sur le fond par l'eau.

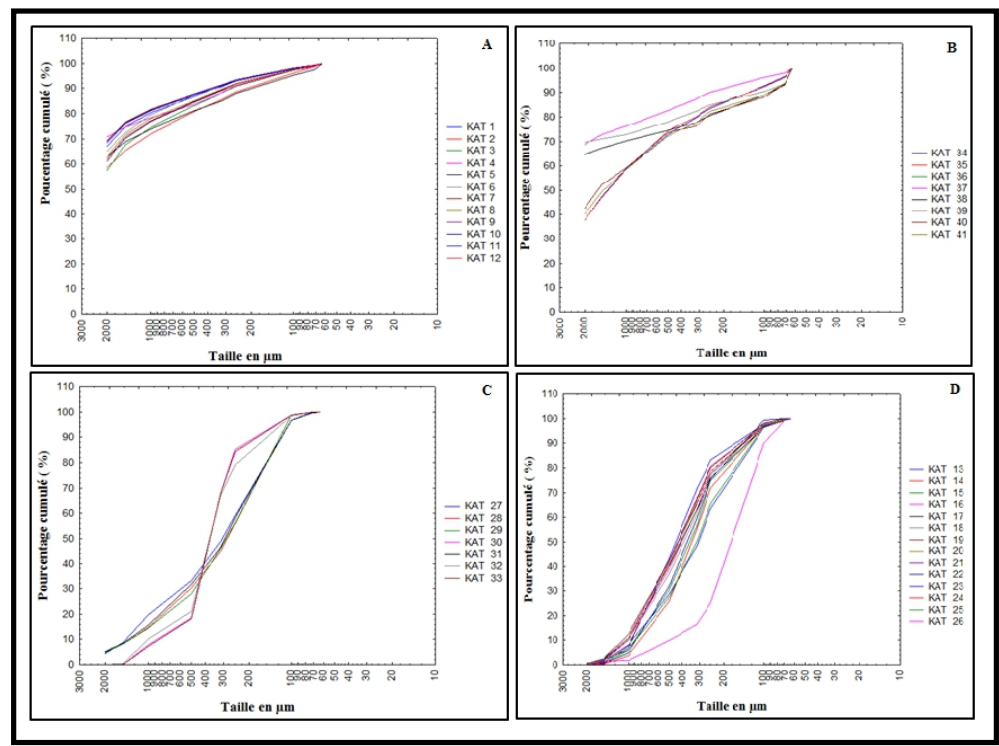

Fig. 9: courbes cumulatives des sables du domaine d'étude ; a-unité VI, b-unité III, c-unité IV, d-unité V 


\section{Paramètres granulométriques}

Les paramètres calculés pour les sables des unités III et VI (Tableau I) indiquent une moyenne comprise entre $-0,49$ et $0,50 \phi$ caractéristiques des sables grossiers. Quant aux unités IV et V leurs moyennes comprises entre 1,13 et 1,56 traduisent des sables moyens. Les écarts-type des différentes unités varient de 1 à 1,79 et évoquent le domaine des sables médiocrement classés.

Les valeurs du skewness des unités III et VI oscillent entre 0,59 et $0,92 \phi$. Elles témoignent d'une courbe asymétrique vers les éléments fins.

Dans l'unité IV, le skewness dont les valeurs varient de - 0,36 à $0,30 \phi$ fait référence à une asymétrie vers les grossiers. Par contre les valeurs du skewness de l'unité $\mathrm{V}$ oscillant entre 0 et $-0,16$ traduisent presque symétrie granulométrique.

Tableau I: Paramètre granulométrique des unités

\begin{tabular}{lccc}
\hline & Grain moyen & Sorting ind ex & \multicolumn{1}{c}{ Skewness } \\
$\begin{array}{l}\text { Unité } \\
\text { lithologique }\end{array}$ & & \\
\hline Unité III & $-0,490,50$ & 1,35 à 1,79 & 0,59 à 0,92 \\
Unité IV & 1,35 à 1,50 & 1,29 à 1,37 & $-0,36$ à $-0,30$ \\
Unité $\mathbf{~}$ & 1,13 à 1,56 & 1 à 1,13 & 0 à $-0,16$ \\
Unité VI & $-0,78$ à 0,39 & 1,03 à 1,45 & 0,84 à 0,90 \\
\hline
\end{tabular}

\section{Mode de Transport des sables}

Le test de VISHER réalisé sur les sédiments superficiels de la zone d'étude donne les résultats présentés par la figure 10 . Il permet de montrer la relation entre la granulométrie des sédiments et leur mode de transport. Ainsi trois populations de sables se distinguent:

a) la population A regroupe les sables transportés par saltation ;

b) la population $\mathrm{B}$ rassemble les sables transportés par suspension ;

c) la population $\mathrm{C}$ est marquée par le transport des grains par roulement.

L'analyse du tableau II, montre que le roulement (C) est le mode de transport dominant des sédiments des unités III $(68,88 \%)$ et VI $(84,58 \%)$. La saltation constitue le mode de transport dominant des sables des unités IV $(64,85 \%)$ et $\mathrm{V}(89,42)$. Le test de Visher appliqué aux sables étudiés, différencie la saltation à une (1), à deux (2) et à trois (3) populations transportés.

La saltation à une population concerne les sédiments de l'unité IV (kat 17, Fig. 10). Celle à deux populations se retrouve au niveau des unités III (kat 35 et 37), IV (kat 27 et 29), V (kat 24 et 25), et VI (kat 1 et 2) (Fig. 10) alors que celle de trois population est rencontrée uniquement dans les 
unités III (kat 32 et 33) et V (kat 21 et 29) (Fig. 10). Cette présence de plusieurs populations transportées par saltation est due au mouvement rythmique des vagues.

Tableau. II: Proportion des sédiments selon le type de transport

\begin{tabular}{lccc}
\hline & Population A & Population B & Population C \\
\hline Type de mouvement & \% Saltation & \% Suspension & \% Roulement \\
Unité III & 27,07 & 4,06 & $\mathbf{6 8 , 8 8}$ \\
Unité IV & $\mathbf{6 4 , 8 5}$ & 0,23 & 34,93 \\
Unité V & $\mathbf{8 9 , 4 2}$ & 0,69 & 9,89 \\
Unité VI & 14,36 & 1,06 & $\mathbf{8 4 , 5 8}$ \\
\hline
\end{tabular}

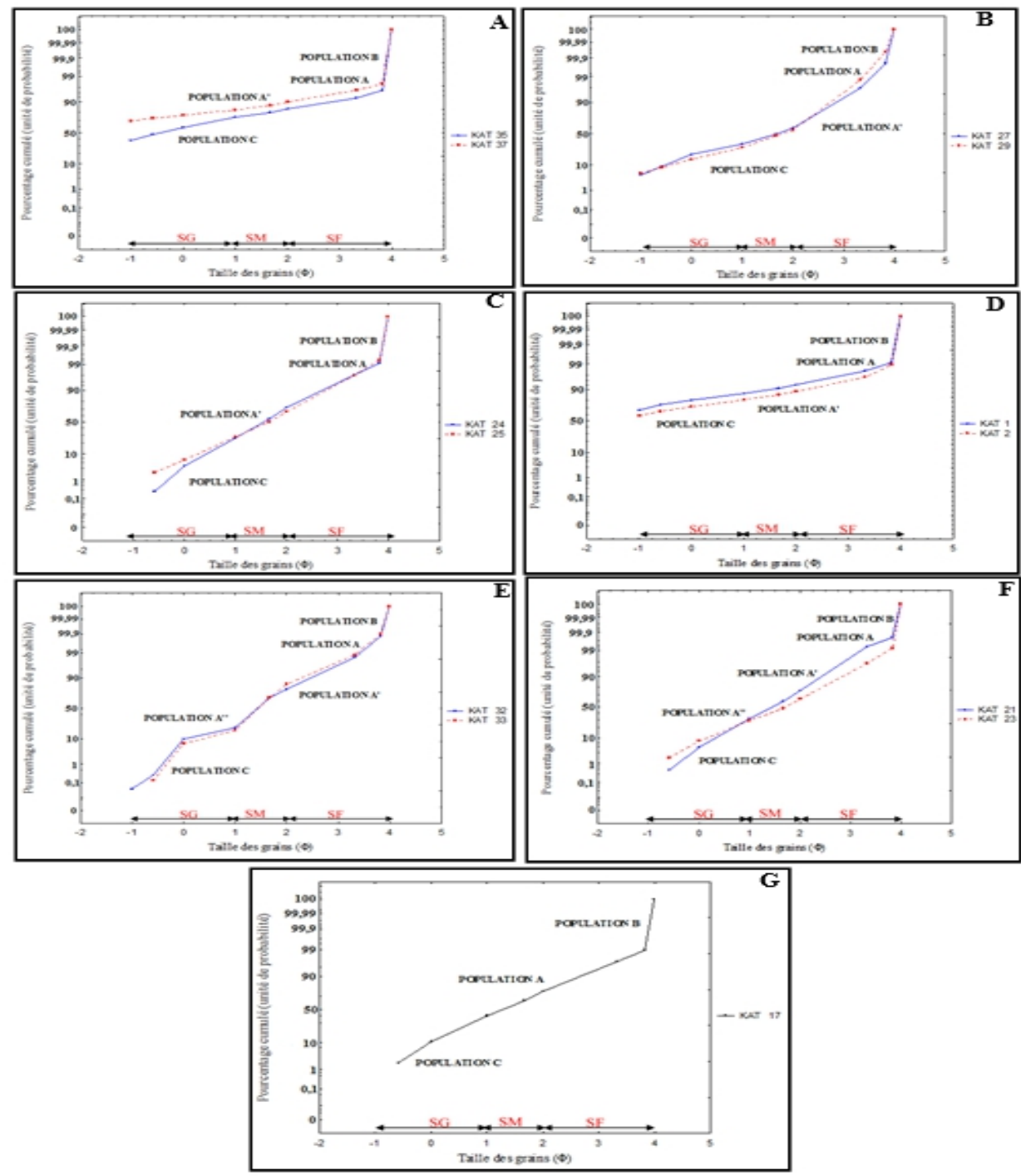

Fig. 10 : Test de Visher des sables à deux populations A; A-unité III, B-IV, C-V, D-VI ; à trois populations $\mathrm{A} ; \mathrm{E}$-unité IV, $\mathrm{F}$-unité $\mathrm{V}$; une population $\mathrm{A} ; \mathrm{G}$ - unité $\mathrm{V}$ 
Par ailleurs, la caractérisation du type de transport dans les différentes classes granulométriques représentée par le tableau III montre que :

a) le mode de transport des sables fins $(\phi<2 \phi)$ dans toutes les unités est dominé par le transport par saltation $(99,23 \%$ en moyenne du matériel fin). Seule une partie infime $(0,77 \%)$ de ces sables fins est portée par suspension.

b) les sables moyens de taille comprise entre 1 et $2 \phi$ se déplacent essentiellement par saltation (89,61\% du matériel moyen). Eventuellement $10,39 \%$ de ces sables moyens est transporté par roulement.

c) le transport par roulement est le mode de transport dominant des sables grossiers $(\phi>1 \phi)$ avec une proportion de 77,68\% du matériel grossier. Par ailleurs $22,32 \%$ de ce matériel grossier est transporté par saltation.

Tableau. III: Caractéristique du type de transport selon

\begin{tabular}{|c|c|c|c|c|}
\hline Unité lithologique & $\begin{array}{c}\text { Classe } \\
\text { granulométrique }\end{array}$ & Saltation \% & Suspension \% & Roulement \% \\
\hline \multirow{3}{*}{ UnitéIII } & Sable grossiers & 18,75 & 0 & 81,25 \\
\hline & Sable moyens & 100 & 0 & 0 \\
\hline & Sable fins & 97,76 & 2,24 & 0 \\
\hline \multirow{3}{*}{ Unité IV } & Sable grossiers & 21,43 & 0 & 78,57 \\
\hline & Sable moyens & 58,44 & 0 & 41,56 \\
\hline & Sable fins & 99,9 & 0,06 & 0 \\
\hline \multirow{3}{*}{ Unité $\mathbf{V}$} & Sable grossiers & 49,12 & 0 & 50,88 \\
\hline & Sable moyens & 100 & 0 & 0 \\
\hline & Sable fins & 99,77 & 0,23 & 0 \\
\hline \multirow{3}{*}{ Unité VI } & Sable grossiers & 0 & 0 & 100 \\
\hline & Sable moyens & 100 & 0 & 0 \\
\hline & Sable fins & 99,45 & 0,55 & 0 \\
\hline
\end{tabular}

\section{Environnement de Dépôt}

Les diagrammes de Moiola et Weiser, appliqué aux sables étudiés révèle deux milieux de dépôt (fig. 11 et 12). Le diagramme So/Md montre que la dispersion se fait à $100 \%$ dans le domaine de rivière. En outre, le diagramme $\mathrm{Md} / \mathrm{Sk}$ montre que la dispersion se fait $100 \%$ dans le domaine dune côtière. L'analyse de ces diagrammes montre donc que les sables du domaine d'étude ont été déposés dans un environnement de dépôt de type rivière et dune côtière. 

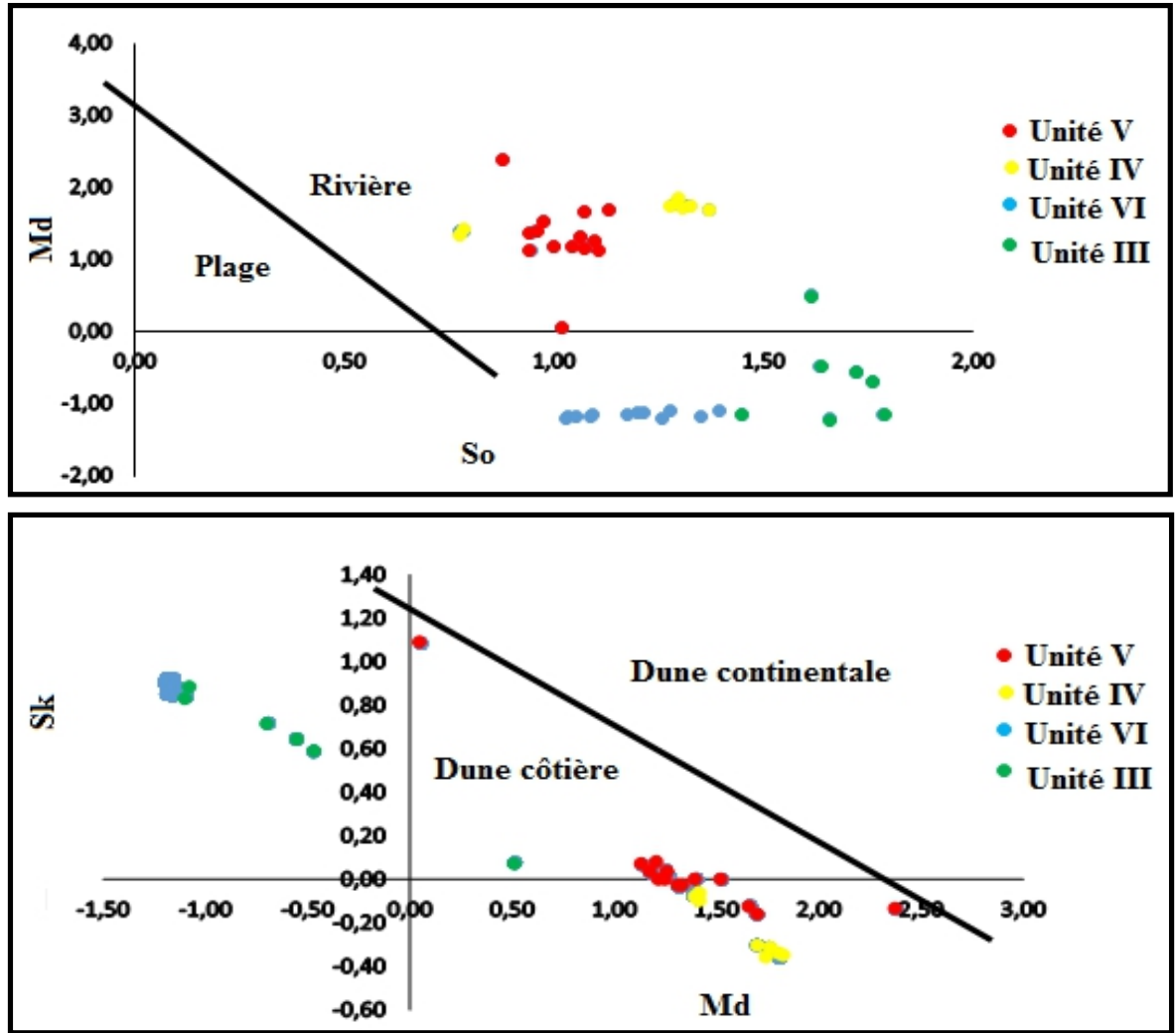

\section{Etude minéralogique}

\section{- Minéralogie des sables}

L'analyse des grains de sable révèle une variété de minéraux (Tableau IV) se rencontre particulièrement dans les fractions fines (unité II, III, IV, V et VI). Le pourcentage de minéraux lourds est élevé dans l'argile silteuse (unité II) par rapport aux autres formations rencontrées. Dans les unités II et III, s'observe une abondance de goethite, d'hématite et de limonite. Par contre, le rutile, l'ilménite et le zircon présentent un fort pourcentage dans les unités IV et V. Néanmoins, l'on note une prédominance des minéraux (Zircon, Rutile, Ilménite, Chlorite, Sphène, Apatite, Tourmaline, Sillimanite, Chromite et Grenat) provenant des roches cristallines, probablement à cause de leur dureté et de leurs résistances à l'altération lors des processus d'érosion et de transport. 
Tableau. IV: Pourcentage relative des minéraux dans les différentes unités lithologique

\begin{tabular}{lccccc}
\hline & Unité II & Unité III & Unité IV & Unité V & Unité VI \\
\hline Géothite & 5,96 & 5,54 & - & - & 3,19 \\
Hématite & 7,95 & 13,23 & - & - & 2,19 \\
Limonite & 5,63 & 5,54 & 0,34 & 0,16 & - \\
Quart & 39,74 & 53,85 & 89,25 & 91,79 & 56,56 \\
Muscovite & 6,23 & - & 0,68 & - & 0,40 \\
Zircon & 0,33 & - & 0,85 & 0,32 & - \\
Rutile & 0,33 & - & 1,19 & - & - \\
Ilménite & 0,99 & 1,23 & 1,19 & 2,90 & 0,2 \\
Chlorite & 0,33 & 0,31 & - & - & - \\
Sphène & 1,32 & 0,92 & - & - & - \\
Apatite & 1,99 & - & 0,68 & - & - \\
Tourmaline & 2,65 & - & 1,88 & 2,25 & - \\
Sillimanite & 0,33 & 4,92 & - & - & 0,6 \\
Feldspath & - & 0,62 & - & 0,64 & - \\
Chromite & - & 3,08 & 0,17 & - & 0,4 \\
Grenat & & & & & \\
Fragment de roches & 16,23 & 10,49 & 3,79 & 1,29 & 33,07 \\
\hline
\end{tabular}

\section{Minéralogie des argiles}

La diffractométrie des argiles (fig. 13) indique six types de minéraux. Le quartz et la kaolinite sont rencontrés dans tous les échantillons. La Goethite et la halloysite (trace) sont absents dans l'unité I contrairement à l'apatite et au gypse qui sont uniquement rencontrés dans ladite unité. 


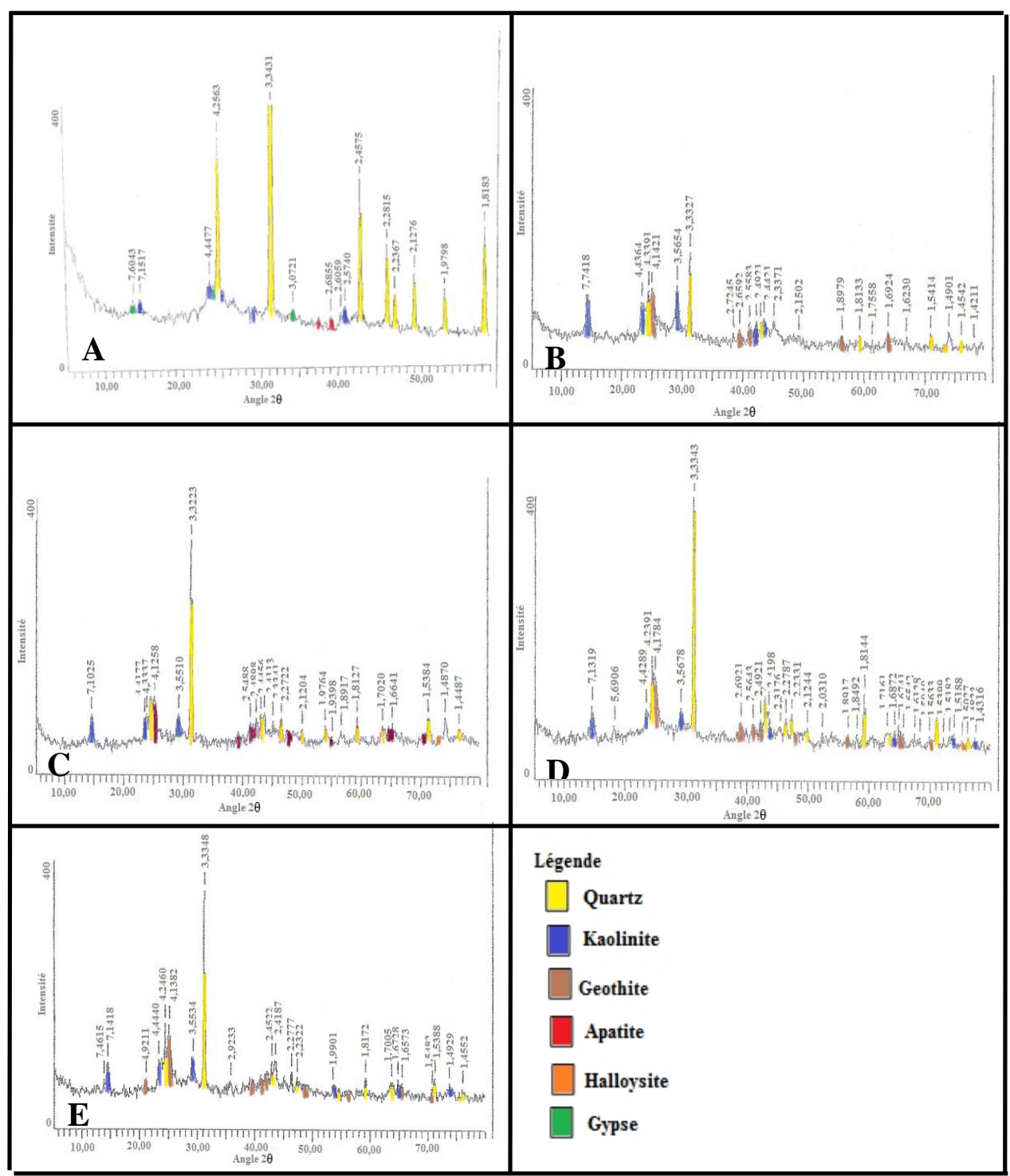

Fig. 13 : Distribution des minéraux de la fraction argileuse les unités I (A), II (B), III (C), $\mathrm{V}(\mathrm{D}), \mathrm{VI}(\mathrm{E})$

\section{Morphoscopie des grains de Quartz}

Les sables observés sont arrondis aux anguleux en passant par les sub-anguleux et les sub-arrondis (Fig. 14). Cette étude révèle que les unités II et III, sont dominées par les formes arrondies et sub-arrondies, évoquant une source pourvoyeuse de clastiques lointaine et un transport relativement long. Par contre les unités I, IV et V montrent une prédominance en grains sub-anguleux, qui traduisent un milieu de dépôt proche de la source génératrice de sables. La plupart des grains rencontrés dans les différentes 
unités présentent un aspect émoussé luisant. Ainsi, le transport des sédiments s'est effectué dans un milieu aqueux. La coloration de certains grains allant du jaune orangé à roux (Figure. 14) est probablement liée à la présence d'oxyde de fer.

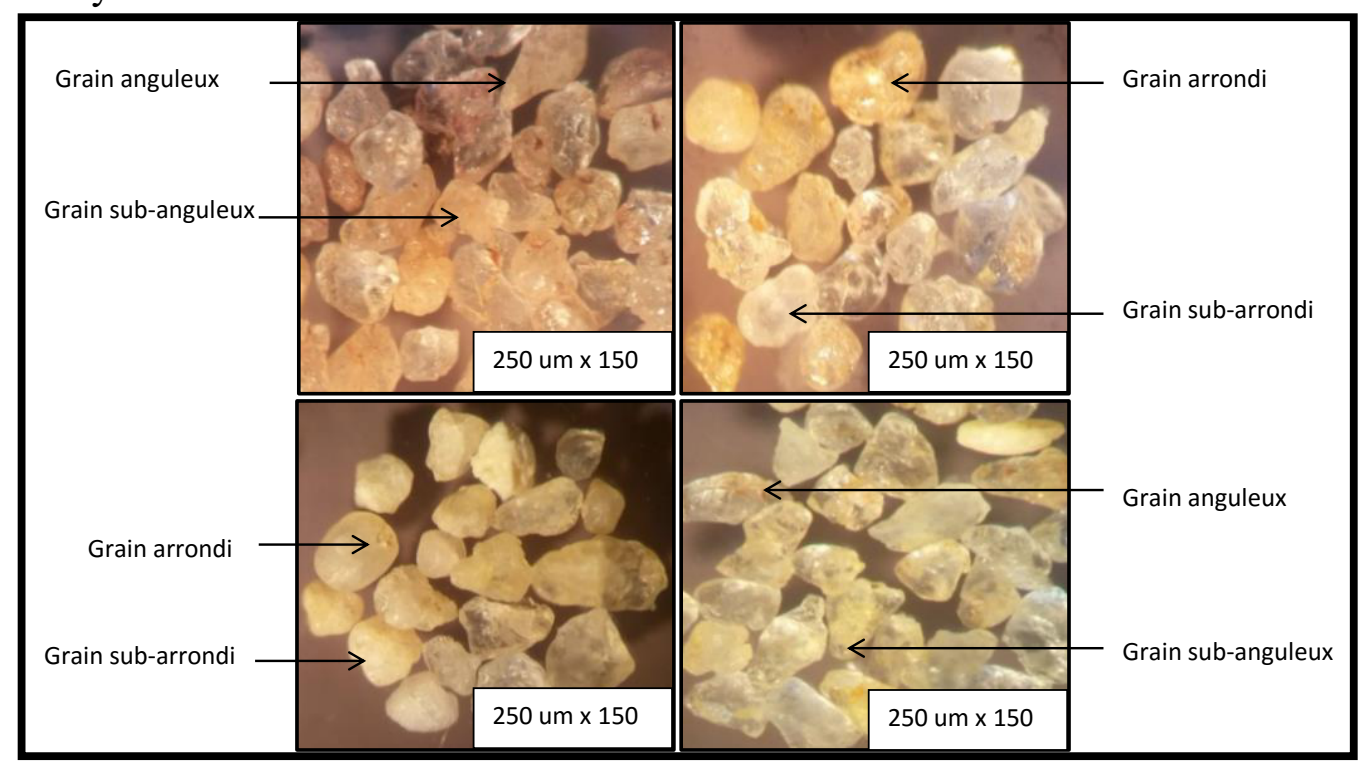

Fig. 14 : Quelques aspects morphoscopiques des grains de quartz issus des unités

\section{Discussion}

La synthèse lithologique des différents sondages étudiés relève que, le domaine d'étude est essentiellement formé d'argile et de sable. Les sédiments argileux sont beaucoup plus localisés au Sud alors que les sables sont au Nord de la zone. La taille des grains diminue du Nord vers le Sud. Cette diminution révèle le sens de l'écoulement Nord-Sud des cours d'eau dans le bassin ivoirien (Assalé, 2013). Les différentes unités définies comportent des couleurs variées; brune à noire, brun-rougeâtre, jaune, bariolée et grise. La couleur noire liée à la richesse en matière organique, est attestée par la présence de débris végétaux (Chevillon, 1992). Celles-ci s'observent principalement dans les puits situés dans les paléochénaux en "V". Les unités rouges, bariolée et brunes, dont la coloration est liée à la présence de pigments d'hématite finement dispersé $\left(\mathrm{Fe}_{2} \mathrm{O}_{3}\right)$, sont d'origine continentale (Launay, 1972, Dugas, 1973; Van Houten, 1973; Einsele, 1992, Eren \& Kadir, 2013). Il existe deux hypothèses pour expliquer l'origine de l'hématite pigmentée: (i) l'hématite est détritiquement dérivée de sols latéritiques (Krynine, 1949 et Folk, 1976, in Eren \& Kadir, 2013 ), tandis que (ii) l'autre authigène c'est à dire post dépôt par l'altération de fer apporté par grains détritiques (Walker, 1967; Eren et Kadir, 1999, in Eren \& Kadir , 2013). Cela traduirait en effet, un paléoclimat tropical semi-aride. Selon 
Chevillon (1992), les teintes grises à noires observées dans l'unité I, pourraient être attribuées à la réduction du fer d'origine terrigène en sulfure de fer. Dans cette unité, s'observe la glauconite et la pyrite évoquant un milieu de dépôt marin peu profond mais peu oxygéné avec un ralentissement de la sédimentation (Odin, 1975, Yao et al, 2011). Les faciès de types sigmoïde et logarithmique ont permis de caractériser les types de dépôt: (i). le faciès logarithmique (Tricart (1965), caractérisent un dépôt par excès de charge lors de la diminution de la vitesse de transport dans les milieux calmes. La forme en S qui traduit un " faciès de dépôt par excès de charge » est un faciès de sable littoral où les grains sont transportés par saltation (Pinot 1994). Pour Saidi et al. (2004) et Yao (2012), ce faciès indique des sables bien classés dans un milieu peu agité avec évacuation de particules fines. Le test de Visher (1969) indique que le transport des sédiments étudiés est assuré par saltation et par roulement. Le texte de Vischer (1969) appliqué aux sables étudiés différencie des saltations à deux et à trois populations transportées.

La présence de deux à trois populations transportés par saltation selon Visher (1969) est caractéristique du mouvement alternatif des vagues. Ce mouvement est sensible en milieu littoral dans les zones de déferlement des vagues et en milieu deltaïque à la limite de la ligne de rivage. Les grains de quartz de ces sables sont sub-arrondis, arrondis à sub-anguleux rarement anguleux. Ils présentent un aspect émoussé luisant de couleur gris à jaune orangé traduisant un transport en milieux aquatiques continentaux ou d'évolution en milieux marins (Cailleux, 1947 in Saaidi, 1991). Selon Martin (1973), la couleur jaune orangé à roux des grains seraient liée à la présence d'oxyde de fer. La prédominance des minéraux (Zircon, Rutile, Ilménite, Chlorite, Sphène, Apatite, Tourmaline, Sillimanite, Chromite et Grenat) provenant des roches cristallines, est probablement liée à leurs duretés et leurs résistances à l'altération lors des processus d'érosion et de transport. Par ailleurs, la présence de ses minéraux pourrait témoigner de l'origine proximale des sédiments. L'abondance relative quartz par rapport aux autres minéraux serait due à leur résistance à l'altération météoritique, et à se concentrer dans les sédiments transportés par les rivières sous climat tropical (Johnsson et al., 1996). La présente des oxydes et hydroxydes tels que la limonite, l'hématite et la goethite, un milieu de de dépôt de type continental (Boga, 2015). L'analyse diffractométrique de la fraction argileuse a mise en évidence différentes minéraux. L'abondance de grains de quartz prouve que la kaolinite présente dans les échantillons étudiés est un minéral d'origine détritique (Mizota et Longstaffe, 1996). Ainsi, la kaolinite provient de l'altération des roches ignées felsiques du socle, sous un climat chaud et humide par monosialllitisation (Pedro, 1966, Nguetnkam J.-P. et al, 2006 ). Ce type d'altération est couramment observé et décrit en milieu 
intertropical (Yongue-Fouateu, 1986 ; Bilong, 1988 ; Nguetnkam et al, 2006). C'est en cela que l'abondance relative de la géothite dans les échantillons étudiés est justifiée.

\section{Conclusion}

L'analyse granulométrique des sédiments de la zone d'Eboinda montre une sédimentation argileuse et sableuse dominante. Elle a révélé six (6) faciès lithologiques : argiles sableuses gravuleuse, sables argileux, argiles sableuses, argiles silteuses, sables et argiles. Les sédiments sableux sont cartographiés au Nord du domaine d'étude, quant aux sédiments argileux au Sud. Les courbes granulométriques cumulatives logarithmique et sigmoïdale traduisent une dynamique également variable. Les sables des unités III et VI à allure de type logarithmique sont accumulés par roulement. Les sables des unités $\mathrm{V}$ et IV à allure sigmoïdale sont dominés par saltation. Les indices granulométriques indiquent généralement un milieu de dépôt de type rivière et dune côtière. Le cortège minéralogique est quasiment dominé par les minéraux lourds (sphène, zircon, tourmaline, ilménite, hématite, apatite, limonite, sillimanite, chromite, grenat, chlorite, rutile, goethite). L'étude morphoscopie des grains de quartz révèle une prédominance des formes arrondies et sub-arrondies suggérant ainsi un transport en milieu aqueux sur une longue distance. Par contre les sables des unités argileuses sont essentiellement sub-anguleux, évoquant une source génératrice proche. L'aspect émoussé luisant de la plupart des grains rencontrés, confirme le mode de transport en milieux aqueux.

\section{Remerciements}

Les auteurs souhaitent remercier les différents responsables de la Direction de la SODEMI qui nous ont permis d'avoir accès aux d'échantillons de forages, enfin de réaliser cet travail.

\section{References :}

AKA K., 1991. La sédimentation quaternaire sur la marge de Côte d'Ivoire : Essai de modélisation. Thèse Doctorat Etat ès Sciences Naturelles. Univ. Abidjan, ${ }^{\circ} 146,320 \mathrm{p}$.

ASSALE F, Y-P., 2013. Caractérisation sédimentologique, palynologique, géochimique et paléoenvironnementale des formations de l'est du bassin onshore de Côte D'Ivoire. Thèse de doctorat, Univ. F.H.B. Cocody, 401p.

BILONG D., 1988. Organisation et évolution d'une couverture ferralitique en zone tropicale

humide (Cameroun). Genèse et transformation d'ensembles ferrugineux indurés profonds. Thèse

Doct, Univ. Poitiers, 164p. 
BOGA. A.H., 2015. Analyse sédimentologie et stratigraphie de sequences sedimentaires ( Crétacé-Quaternaire) de l'Est du bassin sédimentaire de Côte D'Ivoire: Caractérisatio des processus de dépôts détritiques. Thèse de doctorat, Univ. F.H.B. Cocody, 198p.

CAILLEUX A., 1947.Distinction des sables marins et fluviatiles. Bull. Soc. Géol. Fr, 5ème série, $t$. $X V$, pp. 375-404.

CHEVILLON.C., 1992. Biosédimentologie du grand lagon Nord, Etude \&THESE, ORSTOM, Paris, 223p.

CHIERICI A., 1996. Stratigraphie, paléoenvironnements et évolution géologique du bassin de Côte d'Ivoire-Ghana. Géologie de l'Afrique et de l'Atlantique Sud : actes colloques Angers 1994, 117p.

DIAN N., 1977. Rapport de la SODEMI-Abidjan. A : observation dans la région d'Eboïnda-Ebocco.B : observation dans la région de Bonoa-Samo,10 p.

DUGAS F., 1973. La sédimentation dans le sud du lagon de la Nouvelle Calédonie (du Mont Dorè au Canal de la Havannah). Cah. ORSTOM, Sér. Géol., Nouméa : 1-13.

DIOULO L. Y.R., 2009. Etude etude des indices de phosphates d'eboinda (region d'aboisso, sud-est de la côte d'ivoire), DEA, Uni. Abidjan, 48p.

EINSELE. G., 1992. Sedimentary Basins - Evolution, Facies, and Sediment Budget. Berlin: Springer.

EREN - M \& KADIR. S., 2013. Colour origin of red sandstone beds within the Hudai Formation (Early Cambrian), Aydincik (Mersin), southern Turkey FRIEDMAN, G.M., 1961. Distinction between dune beach and river sands from their textural characterestics. Journal of Sedimentary Petrology 31. pp 514-529.

FOLK R.L. and WARD W.C., 1957. Brazos Rivers Bar: a study in the significance of grain size parameters. J. Sed.Petrol., Tulsa (Okl.), 27 (1), 326, 19 p.

FOLK. R. L., 1976. Reddening of desert sandstone: Simpson Desert, N.T., and Australia. J Sediment Petrol 46: 604-615.

JOHNSSON, M. J,, STALLARD, R. F. et MEADE, H. H., 1996. First cycle quartzarenite dans the Orinoco River basin, Venezuela and Columbia. Journal of Geology, 96: 263- 277.

KRYNINE. P.D., 1949. The origin of red beds. Trans NY Acad Sci 11: 6068.

LAUNAY J., 1972. La sédimentation en. baie de Dumbéa (côte ouest de Nouvelle Calédonie). Cah. ORSTOM, Sér. Géol., vol. 4, n $1:$ 25-51.

MARTIN L., 1973. Morphologie, sédimentologie et paléogéographique au quaternaire récent du plateau continental ivoirien. Thèse, Paris, $340 \mathrm{p}$.

MIZOTA, C. LONGSTAFFE, F. J., 1996. Origin of cretaceous and Oligocene kaolinites from 
the Iwaizumi clay deposit, Iwate, Northeastern Japan. Clays and Clay Minerals, 44: 408-416.

MOIOLA.R.J \& WEISER D., 1968. Textureal parameters : an evaluation. Journ. Sedim. Petrol. n³8, pp 45-53.

NGUETNKAM J.-P., YONGUE FOUATEU R., BITOM D., BILONG P. ET VOLKOFF B., 2006. Etude pétrologique d'une formation latéritique sur granite en milieu tropical forestier sud-camerounais (Afrique centrale), Étude et Gestion des Sols, Volume 13, 2,2006 - pages 89 à 102

NORMARK W.R.; HESS G.R.; STOW D.A.V.; BOWEN A.J., 1980. Sediment waves on the Monterey fan levee: a preliminary physica1 interpretation. Marine geology 37, p.1-18.

ODIN. G.S., 1975. De glauconiarum constitutione origine aetateque. Thèse de Doctorat, Pans, 250 pp.

PEDRO G., 1966. Essai sur la caractérisation géochimique des différents processus zonaux

résultant de l'altération des roches superficielles (cycle alumino - silicique). C. R. Acad. Sci.,

262, pp. 1828-1831.

PETROCI et BEICIP. , 1990. Côte d'Ivoire, Evaluation pétrolière (Rapport interne), $68 \mathrm{p}$

PARFENOFF. A, POMEROL. C, TOURENQ. J., 1970. Les minéraux en grains, méthode d'étude et de détermination. Edition Masson, $578 \mathrm{P}$.

PINOT.J.P. 1994. Manipulations sédimentologiques courantes M.W. TURGAL.07.SEDI Edition de février 1994. 118p.

RIVIERE A. 1953. Méthode d'interprétation des granulométries des sédiments meubles. Rev. Inst.Fr. du Pétrole et Annales des combustibles liquides. 1953, 8, p. 102.1952, 61, II. P.155.

SAAIDI E., 1991. Traité de sédimentologie, pétrographie, environnements sédimentaires. Editions Afrique Orient, $399 \mathrm{p}$

SAIDI H., BRAHIM M., et GUEDDARI M., 2004. Caractérisation granulométrique et minéralogique des sédiments de surface de la Frange littorale Sidi Bou Said-la Goulette. Bull Inst. Natn. Scien. Tech. Mer de Salammbô, vol. 31, pp 97-106.

SODEMI., 2011. Côte d'Ivoire, Contrôle du potentiel en phosphate de la Région d'Eboinda (Rapport interne), 20p.

TRICART J., 1965. Principes et méthodes de la géomorphologie, Masson et Cie, Paris, 496p.

VAN HOUTEN. F.B., 1973. Origin of red beds: a review 1961-1972. Annu Rev Earth Planet Sci 1: 39-61.

VISHER.G.S., 1969. Grain size distributions and depositional processes. Journ. Sedim.Petrol., Tulsa, vol. 39, pp. 1074-1106. 
WALKER. T. R., 1967. Formation of red beds in modern and ancient deserts. Geol Soc Am Bull 78: 353-368.

YAO N'GORAN J. P., DIGBEHI ZELI B., MONDE SYLVAIN, KRA AKOUA C., AKA KOUAME, BLEOUE N'ZALASSE, TEA YASSI J. KPLOHI YABA L. H. \& DUFFI KOUAME L., 2011. Etude Sedimentologique et Esquisse Paleoenvironnementale des formations de Fresco, Sciences \& Nature Vol. $8 \mathrm{~N}^{\circ} 1: 73-84$

YAO N. J-P., 2012. Caractérisation sédimentologique, minéralogique, géochimique et biostratigraphique des falaises vives de Fresco : région de Grand-Lahou (Côte d'Ivoire). Thèse de doctorat, Univ. F.H.B. Cocody, $187 \mathrm{p}$.

YONGUE-FOUATEU R., 1986. Contribution à l'étude pétrologique de l'altération et des faciès de cuirassement ferrugineux des gneiss migmatitiques de la région de Yaoundé. Thèse $3^{\mathrm{e}}$ cycle Univ. Yaoundé, 214 p. 